publication of the Synopsis issue of the Bulletin by June 1 should enable all members of committees to have the material for examination at least two months before the opening of the Congress and in time for reasonable advance consideration of the various proposals.

The Editorial Board invites all committee chairmen and secretaries to make full use of the pages of the Bulletin in getting information to all committee members.

\title{
PROVISIONAL PROGRAMME OF MEETINGS OF THE TAXONOMIC SUBCOMMITTEES
}

Permanent Secretary S. T. COWAN has outlined a provisional programme of meetings for the several Taxonomic Subcommittees of the International Committee. It will be noted that one meeting only of each subcommittee is provided. One session may be sufficient for some subcommittees, others will require two or perhaps more. The chairmen of the several subcommittees will be able to arrange for other sessions if needed.

Some of the subcommittees may wish to meet in the week immediately preceding the formal opening of the Congress, as is being done by the Judicial Commission.

The Chairmen of the several Subcommittees should note that all recommendations which require approval of the Plenary Session to become Official Acts of the Congress should be in the hands of one of the Permanent Secretaries, Dr. Cowan or Dr. Wikén, for presentation to the International Committee on Bacteriological Nomenclature at its meeting at 2:30 P.M. (14:30) on Friday, September 11 th.

The times of the meetings provisionally arranged for by the several subcommittees are as follows:

Virus Subcommittee. Chairman Dr. C. H. Andrewes. Time of Meeting, Tuesday Sept. 8, 2:00 p. m. (14:00) 
BACTERIOLOGICAL NOMENCLATURE AND TAXONOMY

Clostridium Subcommittee. Chairman Dr. A. R. Prévot. Time of Meeting, Tuesday Sept. 8, 2:00 p.m.(14:00)

Provisional Staphylococcus Phage-typing Subcommittee. Convener Dr.R.E.O. Williams.

Time of Meeting, Tuesday Sept. 8, 2:00 p.m. (14:00)

Enterobacteriaceae Subcommittee. Co-chairmen Dr.F. Kauffmann and Dr. P.R. Edwards.

Time of Meeting, Tuesday Sept. 8, 2:00 p.m.(14:00)

Leptospira Subcommittee. Chairman Dr. J. W. Wolff.

Time of Meeting, Tuesday Sept. 8, 2:00p.m. (14:00)

Streptococcus Subcommittee. Acting Chairman

Dr. V.D. Allison.

Time of Meeting, Wednesday, Sept. 9, 2:00 p. m. (14:00)

Provisional Streptococcus Serotyping Subcommittee.

Convener Dr.R.E.O.Williams.

Time of meeting, Wednesday, Sept. 9, 2:30 p.m. (14:30)

Medical Mycopathology Subcommittee. Chairman

Dr. W. J. Nickerson.

Time of Meeting, Wednesday, Sept. 9, 2:00 p.m. (14:00)

Mycobacterium Subcommittee. Chairman Dr. P. Hauduroy

Time of Meeting, Wednesday, Sept. 9, 2:00 p.m. (14:00)

Neisseria Subcommittee. Chairman Dr.E. G. D. Murray Time of Meeting, Wednesday, Sept. 9, 2:00 p.m. (14:00) 


\section{SUMMARY PROGRAMME}

Thursday, September 3 rd

09:00

Friday, September 4 th

$09: 00$

Saturday, September 5th

09:00

Sunday, September 6th

$$
10: 00
$$

Monday, September 7 th

$$
\begin{aligned}
& 08: 30-12: 30 \\
& 14: 30 \\
& 15: 30-18: 30
\end{aligned}
$$

Tuesday, September 8 th
- Judicial Commission

- Judicial Commission

- Judicial Commission

$$
\begin{aligned}
& \text { Opening Session } \\
& \text { Afternoon free }
\end{aligned}
$$

- Scientific Sessions

- Nomenclature Committee

- Scientific Sessions

- Scientific Sessions

- Clostridium Subcommittee

- Enterobacteriaceae Subcommittee

- Leptospira Subcommittee

- Staphylococcus phage typing Subcommittee

- Virus Subcommittee

- Scientific Sessions

- Judicial Commission

- Reception at the Campidoglio 
BACTERIOLOGICAL NOMENCLATURE AND TAXONOMY

Wednesday, September 9 th

\begin{tabular}{|c|c|}
\hline $08 \cdot 30-12 \cdot 30$ & - Scientific Sessions \\
\hline $14: 00$ & - Streptococcus Subcom- \\
\hline $14: 00$ & $\begin{array}{l}\text { - Medical mycopathology } \\
\text { Subcommittee }\end{array}$ \\
\hline $14: 00$ & - $\frac{\text { Mycobacterium Subcom- }}{\text { mittee. }}$ \\
\hline $14: 00$ & - Neisseria Subcommittee \\
\hline $14: 30$ & - Judicial Commission \\
\hline $14: 30$ & - Streptococcus serotyping \\
\hline $15: 30-18: 30$ & - Scientific Sessions \\
\hline $21: 00$ & $\begin{array}{l}\text { - Theatre performance or } \\
\text { concert. }\end{array}$ \\
\hline
\end{tabular}

Thursday, September 10th

Excursion

Friday, September 11 th

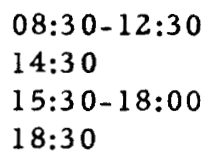

- Scientific Sessions

- Nomenclature Committee

- Scientific Sessions

- Visit to the Instituto Superiore de Sanita and reception.

Saturday, September 12th

$08: 30-12: 30$
$16: 00$
$21: 00$

- Scientific Sessions

- Closing Session

- Official Banquet 